\title{
Asymptotics for $L^{2}$ minimal blow-up solutions of critical nonlinear Schrödinger equation
}

by

\author{
Frank MERLE
}

Université de Cergy-Pontoise, Centre de Mathématiques Avenue du Parc 8, Le Campus, 95033 Cergy-Pontoise, France

ABSTRACT. - In this note, we describe the behavior of a sequence $v_{n}: \mathbb{R}^{N} \rightarrow \mathbb{C}$ minimal in $L^{2}$ such that $\frac{1}{2} \int\left|\nabla v_{n}\right|^{2}-\frac{1}{\frac{4}{N}+2} \int\left|v_{n}\right|^{\frac{4}{N}+2} \leq E_{0}$ and $\left|v_{n}\right|_{H^{1}} \rightarrow+\infty$.

RÉSUMÉ. - Dans cette note, on explicite de façon optimale le comportement d'une suite $v_{n}: \mathbb{R}^{N} \rightarrow \mathbb{C}$ de norme $L^{2}$ minimale telle que $\frac{1}{2} \int\left|\nabla v_{n}\right|^{2}-\frac{1}{\frac{4}{N}+2} \int\left|v_{n}\right|^{\frac{4}{N}+2} \leq E_{0}$ et $\left|v_{n}\right|_{H^{1}} \rightarrow+\infty$.

In the present note, we are interested in the behavior of a sequence $v_{n}: \mathbb{R}^{N} \rightarrow \mathbb{C}$ of $H^{1}$ functions such that

$$
\begin{gathered}
\int\left|v_{n}\right|^{2}=\int Q^{2}, \\
E\left(v_{n}\right)=\frac{1}{2} \int\left|\nabla v_{n}\right|^{2}-\frac{1}{\frac{4}{N}+2} \int\left|v_{n}\right|^{\frac{4}{N}+2} \leq E_{0}, \\
\int\left|\nabla v_{n}\right|^{2} \rightarrow+\infty,
\end{gathered}
$$$$
\text { where } Q \text { is the radial positive symetric solution of the equation }
$$

$$
\Delta v+|v|^{\frac{4}{N}} v=v .
$$


(See references [1], [4], [7] for existence and uniqueness of $Q$.)

This problem is related to the asymptotics of minimal blow-up solutions in $H^{1}$ of the equation

$$
i u_{t}=-\Delta u-k(x)|u|^{\frac{4}{N}} u \text { and } u(0)=\varphi
$$

where

$$
\operatorname{Max}_{x \in \mathbf{R}^{N}} k(x)=1
$$

Indeed, for all $\varphi \in H^{1}$, there is a unique solution in $H^{1}$ on $[0, T]$ ([2], [4]) and

$$
T=+\infty \text { or } \lim _{t \rightarrow T} \int|\nabla u(t, x)|^{2}=+\infty
$$

In addition, $\forall t$

$$
\begin{aligned}
\int|u(t, x)|^{2} d x & =\int|\varphi(x)|^{2} d x \\
E_{k}(u(t)) & =E_{k}(\varphi)
\end{aligned}
$$

where

$$
E_{k}(v)=\frac{1}{2} \int|\nabla v|^{2}-\frac{1}{\frac{4}{N}+2} \int k(x)|v|^{\frac{4}{N}+2} .
$$

From [9]

$$
\forall v \in H^{1}, \quad \frac{1}{\frac{4}{N}+2} \int|v|^{\frac{4}{N}+2} \leq \frac{1}{2}\left(\frac{\int|v|^{2}}{\int|Q|^{2}}\right)^{\frac{2}{N}} \int|\nabla v|^{2}
$$

and it follows from (6)-(9) ([6]) that

$$
\text { if }|\varphi|_{L^{2}}<|Q|_{L^{2}} \text {, then } T=+\infty \text {. }
$$

Moreover under some conditions on $k(x)$, for any $\varepsilon>0$ there are blow-up solutions $u_{\varepsilon}(t)$ such that

$$
\left|u_{\varepsilon}(0)\right|_{L^{2}}^{2}=|\varphi|_{L^{2}}^{2}+\varepsilon \quad([6]) .
$$

Thus the questions are about existence of minimal blow-up solution (that is such that $u(t)$ blows up in finite time and $\int|\varphi|^{2}=\int Q^{2}$ and on the 
form of these solutions. In the case where $k(x) \equiv 1$, the question has been completely solved (see Merle [5]). The general case is still open. We remark that from (6)-(9), if $u(t)$ is a blow up solution, the sequences $v_{n}=u\left(t_{n}\right)$ as $t_{n} \rightarrow T$ satisfies (1)-(3) and we ask about the constrains it imply on $v_{n}$.

The first result in this direction was obtained by Weinstein in [9]. Using the concentration compactness method, he showed that there is a $\theta_{n} \in \mathbb{R}$, $x_{n} \in \mathbb{R}^{N}$ such that

$$
v_{n}=\lambda_{n}^{\frac{N}{2}} e^{i \theta_{n}} Q\left(\lambda_{n}^{\frac{N}{2}}\left(x-x_{n}\right)\right)+\varepsilon_{n},
$$

where

$$
\begin{gathered}
\lambda_{n}=\frac{\left|\nabla v_{n}\right|_{L^{2}}}{|\nabla Q|_{L^{2}}}, \\
\left|\varepsilon_{n}\right|_{L^{2}} \underset{n \rightarrow+\infty}{\longrightarrow} 0 \text { and } \frac{\left|\nabla \varepsilon_{n}\right|_{L^{2}}}{\lambda_{n}} \underset{n \rightarrow+\infty}{\longrightarrow} 0 .
\end{gathered}
$$

In [5], Merle then showed that for all $R>0$, there is a $c>0$ such that

$$
\int_{\left|x-x_{n}\right|>R}\left|\nabla v_{n}\right|^{2} \leq c
$$

We now claim the following result

THEOREM. - Let $\left(v_{n}\right)$ be a sequence of $H^{1}$ functions satisfying (1)-(3) and $\theta_{n}(x)$ be such that $v_{n}=\left|v_{n}\right| e^{i \theta_{n}}$.

i) Phase estimates. There is a $c>0$ such that

$$
\forall n, \quad \int\left|v_{n}\right|^{2}\left|\nabla \theta_{n}\right|^{2} \leq c .
$$

ii) asymptotics on the modulus.

There is a $\varepsilon_{n}(x), x_{n} \in \mathbb{R}^{N}$, and $c>0$ such that

$$
\forall x,\left|v_{n}(x)\right|=\lambda_{n}^{\frac{N}{2}} Q\left(\lambda_{n}\left(x-x_{n}\right)\right)+\varepsilon_{n}(x)
$$

where

$$
\left|\nabla \varepsilon_{n}\right|_{L^{2}} \leq c, \quad\left|\varepsilon_{n}\right|_{L^{2}} \leq \frac{c}{\lambda_{n}} \text { and } \lambda_{n}\left(\frac{|\nabla Q|_{L^{2}}}{\left|\nabla v_{n}\right|_{L^{2}}}\right) \underset{n \rightarrow+\infty}{\longrightarrow} 1 .
$$

Remark. - This Theorem simplifies some proofs in [5], [6]. The case where $v_{n}$ is real valued is also related to similar problems for the generalized Korteweg-de Vries equation with critical nonlinearity. 
Remark. - The Theorem implies in particular for blow-up solution of equation (5) $u(t, x)=|u(t, x)| e^{i \theta(t, x)}$ and $\int|u(t)|^{2}=\int Q^{2}$ the phase gradient is uniformly bounded at the blow-up: there is a $c>0$ such that

$$
\forall 0<t<T, \quad \int|u(t, x)|^{2}|\nabla \theta(t, x)|^{2} d x \leq c .
$$

(Of course, we still have $\left.\int|\nabla| u\right|^{2}(t, x) \underset{t \rightarrow T}{\longrightarrow}+\infty$.)

Remark. - It is easy to check that the result is optimal. We remark that the residual term in the theorem $\varepsilon_{n}=O(1)$ (compared to $o\left(\left|\nabla v_{n}\right|_{L^{2}}\right)$ in [9]).

Proof of the Theorem. - Let $\left(v_{n}\right)$ a sequence of $H^{1}$ function satisfying (1)-(3) and $\theta_{n}(x)$ such that $v_{n}=\left|v_{n}\right| e^{i \theta_{n}}$. We have that

$$
\frac{1}{2} \int\left|\nabla v_{n}\right|^{2}=\frac{1}{2}\left\{\left.|\nabla| v_{n}\right|^{2}+\int\left|v_{n}\right|^{2}\left|\nabla \theta_{n}\right|^{2}\right\}
$$

and

$$
E\left(v_{n}\right)=\frac{1}{2} \int\left|v_{n}\right|^{2}\left|\nabla \theta_{n}\right|^{2}+E\left(\left|v_{n}\right|\right) \text {. }
$$

The idea is to apply the variational identity (9) not with $v_{n}$ but with $\left|v_{n}\right|$.

Indeed, since $v_{n} \in H^{1}$ we have that $\left|v_{n}\right| \in H^{1}$. From (9) (applied with $\left.\left|v_{n}\right|\right)$

$$
\frac{1}{\frac{4}{n}+2} \int\left|v_{n}\right|^{\frac{4}{n}+2} \leq\left.\frac{1}{2}\left(\frac{\int\left|v_{n}\right|^{2}}{\int Q^{2}}\right)^{\frac{2}{N}} \int|\nabla| v_{n}\right|^{2} \leq \frac{1}{2} \int|\nabla| v_{n} \|^{2}
$$

or equivalently

$$
E\left(\left|v_{n}\right|\right) \geq 0
$$

Thus (2), (15), (17) imply that

$$
\frac{1}{2} \int\left|v_{n}\right|^{2}\left|\nabla \theta_{n}\right|^{2} \leq E_{0}
$$

$$
E\left(\left|v_{n}\right|\right) \leq E_{0}
$$

Part i). - It is implied by (18). 
Part ii). - We claim that it is as a consequence of (18)-(19). We prove it in three steps:

- from Weinstein's results, we first obtain rough estimates on $\left|v_{n}\right|$,

- we then choose appropriate approximations parameters,

- we conclude the proof using a convexity property in certain directions of $E$ near $Q$ (and use in a crucial way that $\left|v_{n}\right|$ is a real-valued function).

Step 1: First asymptotics. - Since

$$
\int\left|\nabla v_{n}\right|^{2}=\left.\int|\nabla| v_{n}\right|^{2}+\int\left|v_{n}\right|^{2}\left|\nabla \theta_{n}\right|^{2} \underset{n \rightarrow+\infty}{\longrightarrow}+\infty
$$

and

$$
\forall n, \int\left|v_{n}\right|^{2}\left|\nabla \theta_{n}\right|^{2} \leq c
$$

we have

$$
\int|\nabla| v_{n}||^{2} \underset{n \rightarrow+\infty}{\longrightarrow}+\infty
$$

Moreover,

$$
\int\left|v_{n}\right|^{2}=\int Q^{2} \text { and } E\left(\left|v_{n}\right|\right) \leq E_{0} .
$$

We conclude from Weinstein's result on the existence of $\hat{x}_{n}, \hat{\varepsilon}_{n}$ such that

$$
\left|v_{n}\right|(x)=\hat{\lambda}_{n}^{N / 2} Q\left(\hat{\lambda}_{n} x-\hat{x}_{n}\right)+\hat{\varepsilon}_{n}(x)
$$

where

$$
\begin{gathered}
\hat{\lambda}_{n}=\frac{|\nabla| v_{n} \|_{L^{2}}}{|\nabla Q|_{L^{2}}} \\
\left|\nabla \hat{\varepsilon}_{n}\right|_{L^{2}}=o\left(\hat{\lambda}_{n}\right), \quad\left|\hat{\varepsilon}_{n}\right|_{L^{2}}=o(1) .
\end{gathered}
$$

In order to obtain better estimates on the rest (that is $\left|\nabla \varepsilon_{n}\right|_{L^{2}} \leq c$ ), we have to choose appropriate parameters $\lambda_{n}, x_{n}$ and use the structure of the functional $E(\cdot)$ near $Q$.

Step 2: Choice of the parameters of approximation. - Let us first renormalize the problem. We consider

$$
w_{n, \lambda_{1}, x_{1}}(x)=\left(\frac{\lambda_{1}}{\hat{\lambda}_{n}}\right)^{\frac{N}{2}}\left|v_{n}\right|\left(\left(\lambda_{1} x+\hat{x}_{n}+x_{1}\right) \frac{1}{\hat{\lambda}_{n}}\right)
$$




$$
\tilde{\varepsilon}_{n, \lambda_{1}, x_{1}}(x)=\left(\frac{\lambda_{1}}{\hat{\lambda}_{n}}\right)^{\frac{N}{2}} \hat{\varepsilon}_{n}\left(\left(\lambda_{1} x+\hat{x}_{n}+x_{1}\right) \frac{1}{\hat{\lambda}_{n}}\right) .
$$

We have from (23),

$$
w_{n, \lambda_{1}, x_{1}}(x)=\lambda_{1}^{N / 2} Q\left(\lambda_{1} x+x_{1}\right)+\tilde{\varepsilon}_{n, \lambda_{1}, x_{1}}(x)
$$

where

$$
\frac{\left|\nabla \tilde{\varepsilon}_{n}\right|_{L^{2}}}{\lambda_{1}}+\left|\tilde{\varepsilon}_{n}\right|_{L^{2}} \underset{n \rightarrow+\infty}{\longrightarrow} 0 .
$$

We write (28) as follows

$$
w_{n, \lambda_{1}, x_{1}}(x)=Q(x)+\varepsilon_{n, \lambda_{1}, x_{1}}(x)
$$

where

$$
\varepsilon_{n, \lambda_{1}, x_{1}}(x)=\left[\lambda_{1}^{N / 2} Q\left(\lambda_{1} x+x_{1}\right)-Q(x)\right]+\tilde{\varepsilon}_{n, \lambda_{1}, x_{1}}(x) .
$$

From the implicit function Theorem, we derive easily for $\left|\tilde{\varepsilon}_{n}\right|_{H^{1}}$ small enough the existence of $\lambda_{1, n}, x_{1, n}$ such that

$$
\begin{gathered}
\forall i=1, \ldots, N, \quad \int \varepsilon_{n, \lambda_{1, n}, x_{1, n}} x_{i} Q=0 \\
\int \varepsilon_{n, \lambda_{1, n}, x_{1, n}}|x|^{2} Q=0 .
\end{gathered}
$$

Moreover, from (29)

$$
\left(\lambda_{1, n}, x_{1, n}\right) \underset{n \rightarrow+\infty}{\longrightarrow}(1,0) .
$$

Indeed, let us note

$$
\begin{aligned}
& \text { for } i=1, \ldots, N, \quad \rho_{i}\left(\lambda_{1}, x_{1}\right)=\int \varepsilon_{n, \lambda_{1}, x_{1}} x_{i} Q, \\
& \rho_{N+1}\left(\lambda_{1}, x_{1}\right)=\int \varepsilon_{n, \lambda_{1}, x_{1}}|x|^{2} Q .
\end{aligned}
$$

From (30), we have

$$
\begin{aligned}
& \frac{\partial \varepsilon_{n, 1,0}}{\partial x_{1, i}}=\partial_{i} Q+\partial_{i} \tilde{\varepsilon}_{n, 1,0} \\
& \frac{\partial \varepsilon_{n, 1,0}}{\partial \lambda_{1}}=\frac{N}{2} Q+x . \nabla Q+\left(\frac{N}{2} \tilde{\varepsilon}_{n, 1,0}+x . \nabla \tilde{\varepsilon}_{n, 1,0}\right)
\end{aligned}
$$

where $x_{1}=\left(x_{1,1}, \ldots, x_{1, N}\right)$. 
Therefore, from (29) and integration by parts,

- for $i=1, \ldots, N$, and $j=1, \ldots, N$,

$$
\begin{aligned}
\frac{\partial \rho_{i}}{\partial x_{1, j}}(1,0) & =\int \partial_{j} Q x_{i} Q+o(1)=-2 \delta_{i, j} \int Q^{2}+o(1) \\
\frac{\partial \rho_{i}}{\partial \lambda_{1}}(1,0) & =\int\left(\frac{N}{2} Q+x . \nabla Q\right) x_{i} Q+o(1)=o(1) \\
\frac{\partial \rho_{N+1}}{\partial x_{1, j}}(1,0) & =\int \partial_{j} Q|x|^{2} Q+o(1)=o(1), \\
\frac{\partial \rho_{N+1}}{\partial \lambda_{1}}(1,0) & =\int\left(\frac{N}{2} Q+x . \nabla Q\right)|x|^{2} Q+o(1) \\
& =\frac{N}{2} \int|x|^{2} Q^{2}-\frac{N}{2} \int|x|^{2} Q^{2}-\frac{1}{2} \int x . x Q^{2}+o(1) \\
& =-\frac{1}{2} \int|x|^{2} Q^{2}+o(1) .
\end{aligned}
$$

Therefore, the implicit function theorem implies the existence of $\left(\lambda_{1, n}, x_{1, n}\right)$ such that (31)-(33) hold.

In conclusion, we have proved the following. There exist $\left(\lambda_{1, n}, x_{1, n}\right) \underset{n \rightarrow+\infty}{\longrightarrow}(1,0)$ such that

$$
w_{n, \lambda_{1, n}, x_{1, n}}(x)=Q(x)+\varepsilon_{n, x_{1, n}, x_{1, n}}(x)
$$

where

$$
\forall i=1, \ldots, n, \quad \int \varepsilon_{n, \lambda_{1, n}, x_{1, n}} x_{i} Q=0,
$$

$$
\begin{gathered}
\int \varepsilon_{n, \lambda_{1, n}, x_{1, n}}|x|^{2} Q=0, \\
\left|\varepsilon_{n, \lambda_{1, n}, x_{1, n}}\right|_{H^{1}} \underset{n \rightarrow+\infty}{\longrightarrow} 0 .
\end{gathered}
$$

We now note

$$
\begin{aligned}
w_{n} & =w_{n, \lambda_{1, n}, x_{1, n}} \\
\varepsilon_{n} & =\varepsilon_{n, \lambda_{1, n}, x_{1, n}} .
\end{aligned}
$$

Step 3 : Conclusion of the proof. - Geometry of energy functions at $Q$. Vol. 13, $\mathrm{n}^{\circ} 5-1996$. 
We now use convexity properties of a functional (related to $E$ ) and the fact $\int w_{n}^{2}=\int Q^{2}$ to conclude the proof. Let

$$
H(v)=\frac{1}{2} \int|\nabla v|^{2}-\frac{1}{\frac{4}{N}+2} \int|v|^{\frac{4}{N}+2}+\frac{1}{2} \int v^{2}=E(v)+\frac{1}{2} \int v^{2},
$$

and

$$
H_{2}(v)=\frac{1}{2} \int|\nabla v|^{2}-\frac{4}{N} \int Q^{\frac{4}{N}} v^{2}+\frac{1}{2} \int v^{2} .
$$

We know that $Q$ is a critical point of $H$, and $H_{2}$ is the quadratic part of $H$ near $Q$ (where $v$ is real-valued). Moreover, it is classical that for $|\varepsilon|_{H^{1}} \leq 1$,

$$
H(Q+\varepsilon)-H(Q)=H_{2}(\varepsilon)+\tilde{H}_{2}(\varepsilon)
$$

where $\left|\tilde{H}_{2}(\varepsilon)\right|=o\left(|\varepsilon|_{H^{1}}^{2}\right)$.

From a result of Weinstein [8] (see also Kwong [4]), we have the following convexity property of $\mathrm{H}_{2}$ at $Q$.

Proposition 1. See [8]. - (Directions of convexity of $H$ at $Q$ in the set of real-valued functions.)

There is a constant $c_{1}>0$ such that $\forall \varepsilon \in H^{1}$

If

$$
\forall i=1, \ldots, N, \quad \int \varepsilon x_{i} Q=0
$$

$$
\int \varepsilon|x|^{2} Q=0
$$

$$
\int \varepsilon Q=0
$$

then

$$
H_{2}(\varepsilon) \geq c_{1}\left(\int|\nabla \varepsilon|^{2}+\varepsilon^{2}\right)=c_{1}|\varepsilon|_{H^{1}}^{2} .
$$

Remark. - We have here a strict convexity property (up to the invariance of the equation) except in the direction $Q$ which is not true for the quadratic part of $H$ for complex valued functions (see [8]).

Remark. - This proposition is optimal. Other functions can be chosen also.

Using now crucially estimates on the $L^{2}$ norm, we obtain the following 
Proposition 2 (Control of the $Q$ direction by the $L^{2}$ norm).

Assume

$$
\forall i=1, \ldots, N, \quad \int \varepsilon x_{i} Q=0,
$$

$$
\begin{gathered}
\int \varepsilon|x|^{2} Q=0, \\
\int(Q+\varepsilon)^{2}=\int Q^{2},
\end{gathered}
$$

then there are $c_{1}>0$ and $c_{2}>0$ such that

$$
|\nabla \varepsilon|_{L^{2}}+|\varepsilon|_{L^{2}} \leq c_{2} \text { implies } H_{2}(\varepsilon) \geq c_{1}\left(|\nabla \varepsilon|_{L^{2}}^{2}+|\varepsilon|_{L^{2}}^{2}\right) \text {. }
$$

Remark. - We need control on 3 directions to obtain estimates on $|\varepsilon|_{H^{1}}$ with $\mathrm{H}_{2}(Q+\varepsilon)$. Two directions can be controlled using the invariance of the equation. The last one is controlled by the condition of minimality on the $L^{2}$ norm (among sequence satisfying (2)).

Proof of Proposition 2. - Let us note

$$
\tilde{H}_{2}\left(v_{1}, v_{2}\right)=\frac{1}{2} \int \nabla v_{1} \nabla v_{2}-\frac{4}{N} \int Q^{\frac{4}{N}} v_{1} v_{2}+\frac{1}{2} \int v_{1} v_{2} .
$$

We can write

$$
\varepsilon=z+a Q+b|x|^{2} Q
$$

with

$$
\int z Q=\int z x_{i} Q=\int z|x|^{2} Q=0 \text { for } i=1, \ldots, N .
$$

Indeed $a$ and $b$ have to satisfy

$$
\begin{aligned}
& \int \varepsilon Q=a \int Q^{2}+b \int|x|^{2} Q^{2} \\
& o=a \int|x|^{2} Q^{2}+b \int|x|^{4} Q^{2}
\end{aligned}
$$

Vol. 13, $\mathrm{n}^{\circ}$ 5-1996. 
or equivalently

$$
\begin{gathered}
b=-a\left(\frac{\int|x|^{2} Q^{2}}{\int|x|^{4} Q^{2}}\right) \\
a\left(\frac{\int Q^{2} \int|x|^{4} Q^{2}-\left(\int|x|^{2} Q^{2}\right)^{2}}{\int|x|^{4} Q^{2}}\right)=\int \varepsilon Q
\end{gathered}
$$

(which has always a solution since from the Schwarz inequality and the fact $\left.|x|^{2} Q \neq Q, \int|x|^{2} Q^{2}<\left(\int Q^{2} \int|x|^{4} Q^{4}\right)^{1 / 2}\right)$.

On the other hand, we have from $\int(Q+\varepsilon)^{2}=\int Q^{2}$

$$
\begin{aligned}
& 2 \int Q \varepsilon=-\int \varepsilon^{2} \\
& 2\left(a \int Q^{2}+b \int|x|^{2} Q^{2}\right)=-\int \varepsilon^{2} \\
& 2 a\left(\frac{\int Q^{2} \int|x|^{4} Q^{2}-\left(\int|x|^{2} Q^{2}\right)^{2}}{\int|x|^{4} Q^{2}}\right)=-\int z^{2}+O\left(a^{2}+b^{2}\right)
\end{aligned}
$$

or equivalently,

$$
a c_{0}=-\int z^{2}+O\left(a^{2}\right) \text { where } c_{0} \neq 0
$$

which implies that

$$
a=O\left(\int z^{2}\right) \text { and } b=O\left(\int z^{2}\right)
$$

and for $|\varepsilon|_{H^{1}}$ small enough

$$
|\varepsilon|_{H^{1}}^{2} \geq|z|_{H^{1}}^{2} \geq \frac{1}{2}|\varepsilon|_{H^{1}}^{2}
$$

On the other hand, by bilinearity and Proposition 1, we have for $|\varepsilon|_{H^{1}}$ small enough

$$
\begin{aligned}
H_{2}(\varepsilon)=H_{2}(z) & +2 a \tilde{H}_{2}(z, Q)+2 b \tilde{H}_{2}\left(z,|x|^{2} Q\right)+2 a b \tilde{H}_{2}\left(Q,|x|^{2} Q\right) \\
& +a^{2} H_{2}(Q)+b^{2} H_{2}\left(|x|^{2} Q\right) \\
& \geq H_{2}(z)-c\left(|z|_{H^{1}}(|a|+|b|)+a^{2}+b^{2}\right) \\
& \geq H_{2}(z)-c\left(|z|_{H^{1}}^{3}+|z|_{H^{1}}^{4}\right) \\
& \geq c_{1}\left(|z|_{H^{1}}^{2}\right)-c\left(|z|_{H^{1}}+|z|_{H^{1}}^{4}\right) \\
& \geq \frac{c_{1}}{2}|z|_{H^{1}}^{2} \\
& \geq \frac{c_{1}}{4}|\varepsilon|_{H^{1}}^{2} .
\end{aligned}
$$


This concludes the proof of Proposition 2.

As a corollary of Proposition 2 and (38), we have

COROLlARY. - There are $c_{1}>0$ and $c_{2}>0$ such that if

$$
\forall i=1, \ldots, N, \quad \int \varepsilon x_{i} Q=0
$$

$$
\begin{gathered}
\int \varepsilon|x|^{2} Q=0 \\
\int(Q+\varepsilon)^{2}=\int Q^{2} \\
|\nabla \varepsilon|_{L^{2}}+|\varepsilon|_{L^{2}} \leq c_{2}
\end{gathered}
$$

then

$$
H(Q+\varepsilon)-H(Q) \geq c_{1}\left(\int \nabla \varepsilon^{2}+\int \varepsilon^{2}\right) .
$$

We now apply the corollary. If $w_{n}=Q+\varepsilon_{n}$, we have $-\left|\varepsilon_{n}\right|_{H^{1}} \underset{n \rightarrow+\infty}{\longrightarrow}$, and in particular there is $n_{0}$ such that

$$
\forall n \geq n_{0}, \quad\left|\varepsilon_{n}\right|_{H^{1}} \leq c_{2},
$$

- $\forall i=1, \ldots, N, \int \varepsilon_{n} x_{i} Q=0$.

In addition,

$$
\begin{aligned}
H(Q) & =\frac{1}{2} \int|\nabla Q|^{2}-\frac{1}{\frac{4}{N}+2} \int|Q|^{\frac{4}{N}+2}+\frac{1}{2} \int Q^{2} \\
& =E(Q)+\frac{1}{2} \int Q^{2} \\
& =\frac{1}{2} \int Q^{2}
\end{aligned}
$$

(since the Pohozaev identity for equation (4) yields $E(Q)=0$ ), and

$$
\begin{aligned}
H\left(Q+\varepsilon_{n}\right) & =H\left(w_{n}\right)=H\left(\left(\frac{\lambda_{1}}{\hat{\lambda}_{n}}\right)^{\frac{N}{2}}\left|v_{n}\right|\left(\frac{x \lambda_{1}}{\hat{\lambda}_{n}}+\hat{x}_{n}+x_{1}\right)\right) \\
& =E\left(\left(\frac{\lambda_{1}}{\hat{\lambda}_{n}}\right)^{\frac{N}{2}}\left|v_{n}\right|\left(\frac{x \lambda_{1}}{\hat{\lambda}_{n}}\right)\right)+\frac{1}{2} \int\left|v_{n}\right|^{2} \\
& =\left(\frac{\lambda_{1}}{\hat{\lambda}_{n}}\right)^{2} E\left(\left|v_{n}\right|\right)+\frac{1}{2} \int Q^{2} .
\end{aligned}
$$

Vol. 13, $\mathrm{n}^{\circ} 5-1996$. 
Therefore $\forall n \geq n_{0}$

$$
\left(\frac{\lambda_{1}}{\hat{\lambda}_{n}}\right)^{2} E\left(\left|v_{n}\right|\right)>c_{3}\left(\left|\varepsilon_{n}\right|_{H^{1}}^{2}\right)
$$

or equivalently from (19), (24) and the fact that $\lambda_{1} \rightarrow 1$,

$$
\left|\varepsilon_{n}\right|_{H^{1}}^{2} \leq \frac{c}{2} \frac{1}{\left.\int|\nabla| v_{n}\right|^{2}} \leq c \frac{1}{\int\left|\nabla v_{n}\right|^{2}}
$$

where $c$ is independent of $n$. Thus,

$$
w_{n}=Q+\varepsilon_{n},
$$

with

$$
\left|\varepsilon_{n}\right|_{H^{1}}^{2} \leq \frac{c}{\int\left|\nabla v_{n}\right|^{2}}
$$

Therefore from (26), there is $x_{n}$ such that

(43) $\left|v_{n}\right|(x)=\left(\frac{\hat{\lambda}_{n}}{\lambda_{1}}\right)^{\frac{N}{2}} Q\left(x\left(\frac{\hat{\lambda}_{n}}{\lambda_{1}}\right)+x_{n}\right)+\left(\frac{\hat{\lambda}_{n}}{\lambda_{1}}\right)^{\frac{N}{2}} \varepsilon_{n}\left(\frac{\hat{\lambda}_{n}}{\lambda_{1}} x+x_{n}\right)$.

We remark that from (19), (42), the fact that $\lambda_{1} \rightarrow 1$,

$$
\begin{aligned}
& \frac{\hat{\lambda}_{n}}{\lambda_{1}} \frac{1}{\left(\int \nabla v_{n}^{2}\right)^{\frac{1}{2}}}=\frac{1}{\lambda_{1}}\left(\frac{\left.\int|\nabla| v_{n}\right|^{2}}{\int\left|\nabla v_{n}\right|^{2} \int|\nabla Q|^{2}}\right)^{\frac{1}{2}} \underset{n \rightarrow+\infty}{\longrightarrow} 1 \\
& \left|\left(\frac{\hat{\lambda}_{n}}{\lambda_{1}}\right)^{\frac{N}{2}} \varepsilon_{n}\left(\frac{\hat{\lambda}_{n}}{\lambda_{1}} x+x_{n}\right)\right|_{L^{2}}^{2}=\left|\varepsilon_{n}\right|_{L^{2}}^{2} \leq \frac{c}{\int\left|\nabla v_{n}\right|^{2}} \\
& \left|\nabla\left(\frac{\hat{\lambda}_{n}}{\lambda_{1}}\right)^{\frac{N}{2}} \varepsilon_{n}\left(\frac{\hat{\lambda}_{n}}{\lambda_{1}} x+x_{n}\right)\right|_{L^{2}}^{2} \leq c\left(\frac{\hat{\lambda}_{n}^{2}}{\int\left|\nabla v_{n}\right|^{2}}\right) \leq c
\end{aligned}
$$

conclude the proof of the Theorem. 


\section{REFERENCES}

[1] H. BERESTYCKI and P. L. LiONS, Nonlinear scalar field equations I. Existence of a ground state; II. Existence of infinitely many solutions, Arch. Rational Mech. Anal., Vol. 82, 1983, pp. 313-375.

[2] J. Ginibre and G. Velo, On a class of nonlinear Schrödinger equations I, II. The Cauchy problem, general case, J. Funct. Anal., Vol. 32, 1979, pp. 1-71.

[3] T. Kato, On nonlinear Schrödinger equations, Ann. Inst. Henri Poincaré, Physique Théorique, Vol. 49, 1987, pp. 113-129.

[4] M. K. Kwong, Uniqueness of positive solutions of $\Delta u-u+u^{p}=0$ in $\mathbb{R}^{N}$, Arch. Rational Mech. Anal., Vol. 105, 1989, pp. 243-266.

[5] F. MERLE, Determination of blow-up solutions with minimal mass for Schrödinger equation with critical power, Duke J., Vol. 69, 1993, pp. 427-454.

[6] F. MERLE, Nonexistence of minimal blow-up solutions of equations $i u_{t}=-\Delta u-$ $k(x)|u|^{4 / N} u$ in $\mathbb{R}^{N}$, preprint.

[7] W. A. Strauss, Existence of solitary waves in higher dimensions, Commun. Math. Phys., Vol. 55, 1977, pp. 149-162.

[8] M. I. WeInSteIN, Modulational stability of ground states of the nonlinear Schrödinger equations, SIAM J. Math. Anal., Vol. 16, 1985, pp. 472-491.

[9] M. I. WEINSTEIN, Nonlinear Schrödinger equations and sharp interpolation estimates, Commun. Math. Phys., Vol. 87, 1983, pp. 567-576.

(Manuscript received October 25, 1994; revised version received January 26, 1995.) 